\title{
Construção e evidências de validade de duas escalas de percepção de desenvolvimento profissional
}

\author{
Luciana Mourão - Universidade Salgado de Oliveira, Rio de Janeiro, Brasil \\ Juliana Barreiros Porto - Universidade de Brasília, Brasilia, Brasil \\ Katia Puente-Palacios - Universidade de Brasilia, Brasilia, Brasil
}

\begin{abstract}
Resumo
O marcado aumento do investimento público e privado feito em ações de treinamento, formação e capacitação evidenciam a centralidade que ações voltadas para o desenvolvimento profissional têm cobrado. Esse desenvolvimento, entretanto, não decorre apenas de ações formais mas também de experiências do cotidiano do trabalho. O objetivo do presente artigo foi desenvolver e identificar as evidências de validade de instrumentos de mensuração de desenvolvimento profissional que focassem tanto na percepção de estado atual de desenvolvimento, como na evolução vivenciada pelo respondente. Para tanto, foram desenvolvidas duas escalas a partir de entrevistas e do referencial teórico que foram testadas em amostra de 674 trabalhadores. Os resultados obtidos na realização das análises fatoriais e de confiabilidade ( $\alpha=0,84$ e 0,92 , respectivamente) revelaram que ambas as medidas constituem ferramentas que permitem a adequada mensuração do desenvolvimento profissional, razão pela qual se constituem em instrumentos úteis de diagnóstico desse atributo, em organizações.

Palavras-chave: Avaliação psicológica, Competência profissional, Desenvolvimento profissional.
\end{abstract}

Evidence of validity of the Perception of Professional Development Scale

\begin{abstract}
The increase of public and private investments in training emphasizes the need for policies in professional development. However, this development does not depend solely on formal actions, but also on daily work experiences. The objective of this study is to develop and identify evidence of validity of an instrument to measure professional development that focuses on the perception of the current development, as well as its evolution experienced by respondents. Thus, two scales were developed based on interviews and theory that had been tested in a sample of 674 professionals. The reliabilities and factor analysis results indicated the scales are adequate for measuring the construct $(\alpha=0.84$ and 0.92 , respectively). In conclusion they constitute useful instruments for measuring professional development in organizations.

Keywords: Psychological assessment, Professional competence, Professional development.
\end{abstract}

Construcción y evidencias de validez de dos escalas de percepción de desarrollo profesional

\section{Resumen}

El destacado aumento de inversión pública y privada en acciones de entrenamiento, formación y capacitación evidencia la centralidad que tienen las acciones vueltas al desarrollo profesional. Ese desarrollo, sin embargo, proviene no sólo de acciones formales, sino también de experiencias del cotidiano del trabajo. El objetivo de esta investigación fue desarrollar e identificar evidencias de validez de instrumentos de medición para el desarrollo profesional que se centrasen tanto en la percepción del estado actual de desarrollo, como en la evolución experimentada por el respondiente. Así fueron construidas dos escalas a partir de entrevistas y del marco teórico que se pusieron a prueba en una muestra de 674 trabajadores. Los resultados obtenidos del análisis factorial y fiabilidad ( $\alpha=0,84$ y 0,92 respectivamente) revelaron que ambas las medidas son herramientas que permiten una medición adecuada del desarrollo profesional, por lo que se constituyen en instrumentos de diagnóstico de ese atributo en las organizaciones.

Palabras clave: Evaluación Psicológica, Competencia profesional, Desarrollo profesional.

\section{Introdução}

No cenário nacional e internacional tem havido grande investimento na educação voltada para o trabalho. Na década de 1970, nos Estados Unidos, as despesas de formação por trabalhador alcançavam a média de cem dólares (SamGnanakkan, 2010), portanto, se considerada a estimativa de 90 milhões de trabalhadores naquele período, chega-se a um investimento anual de 9 bilhões de dólares. No final do século XX a estimativa era de que as organizações estadunidenses gastavam anualmente pelo menos 55 bilhões de dólares em treinamento da força de trabalho (Salas \& Cannon-Bowers, 2001), revelando um considerável incremento. $\mathrm{Na}$ Europa, um estudo com 26 países mostra que aqueles com maior investimento por empregado são Dinamarca, Suécia, Holanda, França, 
Finlândia e Bélgica, com valores que variam de 41 euros anuais (aproximadamente 54 dólares), na Romênia, a 1.132 euros (1.489 dólares, aproximadamente), na Dinamarca (Bassanini, Booth, Brunello, De Paola \& Leuven, 2005). Esse conjunto de dados evidencia a importância dada à formação do trabalhador, ao redor do mundo, apesar das diferenças decorrentes da realidade econômica de cada país.

No Brasil, o crescimento da verba destinada a essa finalidade também é notável, pois enquanto, em 2004, o Governo Federal destinou aproximadamente 3 milhões para pagar a realização de atividades descritas como "treinamento em desenvolvimento profissional e gerencial", em 2011, esse valor foi de 9,5 milhões (Portal da Transparência, 2012). Assim, seria esperado que essa busca de desenvolvimento profissional dos trabalhadores aumentasse também a demanda por pesquisas científicas que permitam compreender melhor esse processo.

Os dados mostram que, no setor público brasileiro, investimentos na área de treinamento, desenvolvimento e educação - TD\&E têm aumentado nos últimos anos, porém ainda há muitas lacunas na literatura científica nacional $\mathrm{e}$ internacional sobre o desenvolvimento profissional de trabalhadores. Embora haja grande concentração de pesquisas sobre o impacto do treinamento, este tem sido medido como o resultado de ações específicas de capacitação, ou seja, o quanto o trabalhador treinado está aplicando no trabalho aquilo que aprendeu em determinada ação de capacitação (Mourão \& Meneses, 2012; Freitas \& Mourão, 2012). Entretanto, avaliações de amplo escopo não têm sido realizadas e a investigação do desenvolvimento profissional dos trabalhadores em longo prazo assim como dos aspectos que favorecem esse desenvolvimento permanece como lacuna.

Em temas correlatos, há pesquisas sobre desenvolvimento de carreiras e o entendimento de que estas não estão circunscritas a uma única organização. A literatura da área menciona outros tipos de carreira como a "carreira sem fronteiras", caracterizada pela pluralidade de contextos de trabalho e o desenvolvimento de competências (Arthur \& Rousseau, 1996), a Carreira Proteana que tem a mudança como constante, possibilitando uma variedade de experiências e ao mesmo tempo exigindo adaptabilidade e resiliência (Hall, 2002), ou ainda, a Carreira Multidirecional, caracterizada pela não linearidade das experiências de trabalho, o contrato psicológico transacional e comprometimentos múltiplos e não apenas com a organização (Baruch, 2006).
Embora com alguma semelhança em relação ao conceito de desenvolvimento de carreiras, o conceito de desenvolvimento profissional refere-se especificamente ao processo de aquisição e aperfeiçoamento de conhecimentos, habilidades e atitudes que favorecem o desempenho no trabalho e o avanço individual na carreira. Portanto, nesse conceito não estão abrangidos todos os aspectos do desenvolvimento na carreira como identidade com a profissão, tipos de comprometimentos ou vivência psicológica.

O foco do conceito de desenvolvimento profissional está em uma combinação de processos, que envolvem um conjunto de modalidades de aprendizagem, formais e informais, com enfoque tanto em aspectos cognitivos como também comportamentais e afetivos. O desenvolvimento profissional está diretamente relacionado com as atividades do dia a dia do profissional e deve fazer parte de um processo mais amplo de aprendizagem contínua (Guskey, 2000).

O desenvolvimento profissional consiste, portanto, em uma decorrência natural das ações formais de capacitação, mas não depende apenas das mesmas, estando, ao contrário, vinculado a qualquer tipo de aprendizagem formal ou informal voltada para o ambiente de trabalho. No mundo anglo-saxão, contudo, o conceito de professional development costuma ser utilizado para designar as atividades de aprendizagem induzida, sem uma visão mais ampla de desenvolvimento de competências (Day, 2004). Essa é uma visão restritiva do conceito, pois desenvolvimento profissional corresponde ao crescimento e amadurecimento dos conhecimentos, habilidades e atitudes adquiridos ao longo da vida dos trabalhadores, como resultado de ações formais e informais de aprendizagem no trabalho (Paquay, Wouters \& van Nieuwenhoven, 2012).

Diante do exposto, a temática desenvolvimento profissional circunscreve-se nas práticas de gestão de pessoas e em características pessoais dos trabalhadores. Mas em que pese a importância do desenvolvimento profissional para a melhoria do desempenho da mão de obra e da qualidade da inserção no mundo do trabalho, pouca atenção tem sido dada ao construto. As organizações, em geral, têm dedicado maior atenção à aprendizagem formal de aplicação imediata, em detrimento tanto dos resultados das ações de aprendizagem informal como dos resultados de longo prazo em termos de desenvolvimento dos trabalhadores (Coelho Jr. \& Mourão, 2011).

No que diz respeito à aprendizagem induzida, observam-se algumas tendências para a área tais como: 
(i) o crescente reconhecimento da contribuição do treinamento para as estratégias organizacionais (Butteriss, 1999; Salas \& Cannon-Bowers, 2001; SamGnanakkan, 2010); (ii) a expansão de atuação da área que deixa de ser apenas do interesse das organizações, caracterizando-se o desenvolvimento profissional como uma demanda social (Goldstein \& Gilliam, 1990; Mourão, 2009; Rosseau, 1997); (iii) o aumento da velocidade com que informações tornam-se obsoletas e a maior complexidade cognitiva no trabalho, com qualificação e requalificação mais intensas e rápidas (Aguinis \& Kraiger, 2009; Mourão, 2009); e (iv) o crescimento da educação a distância (Abbad, Zerbini \& Souza, 2010; Salas \& Cannon-Bowers, 2001).

As ações de aprendizagem no trabalho, sejam elas de natureza formal (induzida), por meio dos programas de TD\&E, ou informal (não sistematizada, espontânea, natural) passaram a ter importância estratégica para as organizações (Coelho Jr. \& Borges-Andrade, 2008). Tanto uma como outra contribuem para o aumento de expertise dos trabalhadores, o que está associado ao desenvolvimento profissional dos mesmos (Paquay e colaboradores, 2012).

As ações informais de aprendizagem humana no trabalho dizem respeito à busca por novos conhecimentos e habilidades pelo indivíduo normalmente considerando alguma demanda ou necessidade associada as suas tarefas e podem ter grande relevância para os trabalhadores e para as organizações (Conlon, 2004). Elas acontecem fora dos currículos que constituem programas educacionais (Livingstone, 1999) com base em experiências planejadas ou não (Watkins \& Marsick, 1992), e podem ocorrer a partir de uma dúvida sanada por algum colega mais experiente, pela leitura de manuais e documentos da organização, por meio de observação cotidiana, etc. Assim, o local de trabalho pode ser considerado um espaço de produção contínua do conhecimento coletivo com um processo de aprendizagem informal que ocorre paralelamente às ações de TD\&E (Flach \& Antonello, 2011).

Embora a aprendizagem formal venha recebendo maior ênfase (Malcolm, Hodkonson \& Colley, 2003), é preciso frisar que não há um posicionamento antagônico dos tipos de aprendizagem. Muito pelo contrário, elas devem ser compreendidas como processos articulados que contribuem para o desenvolvimento profissional dos trabalhadores (Antonello, 2005). Mas, possivelmente em função dessa separação encontrada na literatura entre aprendizagem formal e informal, há poucas pesquisas que focalizam o desenvolvimento profissional de forma mais ampla. Há muitos estudos sobre a temática da aprendizagem formal (Aguinis \& Kraiger, 2009; Berkvens, Kalyanpur, Kuiper \& van den Akker, 2012; Golding \& Gray, 2006; Jasper, 2006), mas tanto na literatura nacional como na estrangeira não foram localizadas escalas que permitam mensurar o grau de desenvolvimento profissional em que se encontram os trabalhadores.

Uma vez identificada a importância dos estudos sobre desenvolvimento profissional, ressalta-se a necessidade de construção de instrumentos de medidas válidas e confiáveis que permitam o avanço dos estudos sobre o tema. Assim, é objetivo deste artigo descrever o processo de construção de duas escalas de percepção de desenvolvimento profissional e apresentar evidências de validade e precisão das medidas.

\section{Método}

Neste estudo foram desenvolvidas duas escalas de mensuração com objetivos diferentes, mas tendo o mesmo construto como base - desenvolvimento profissional. $\mathrm{O}$ trabalho envolveu duas etapas sucessivas: o desenvolvimento de duas escalas e, posteriormente, a verificação das propriedades psicométricas das mesmas. Assim, será inicialmente descrito o processo de desenvolvimento das medidas e em seguida o método adotado para a verificação de suas propriedades.

\section{Etapa 1 - Construção dos Instrumentos}

Conforme relatado, foram desenvolvidas duas escalas, a primeira visando avaliar o estado atual de desenvolvimento profissional e a segunda a percepção evolutiva desse desenvolvimento resultante da comparação entre o momento atual e o momento de ingresso no mundo do trabalho. A decisão pelo desenvolvimento de duas medidas foi tomada pela constatação de que, em alguns casos, o foco da avaliação pode estar em mensurar o grau de desenvolvimento profissional que a pessoa percebe ter em termos absolutos (visão atual do desenvolvimento profissional) e, em outros casos, o interesse pode estar na percepção relativa desse desenvolvimento profissional ou mudança vivenciada ao longo do tempo (visão evolutiva).

A definição teórica do construto serviu de base para a definição de propriedades do construto que deveriam ser abordadas pelos itens a serem construídos. A redação dos itens foi extraída da análise do corpo discursivo levantado mediante a realização de entrevistas em profundidade com 10 trabalhadores de 
diferentes setores de organizações públicas e privadas. A amostra para as entrevistas contemplou 5 mulheres e 5 homens, com idades entre 25 e 58 anos e escolaridade variando de ensino médio completo a pós-graduação. Os setores de trabalho dessas pessoas foram diversificados contemplando profissionais que atuam em áreas como comércio, saúde, educação, segurança pública, serviços administrativos e indústria de produtos têxteis. Com base em tais entrevistas foi feita análise de conteúdo categorial (Bardin, 1977; Bauer, 2010), na qual foram identificadas categorias temáticas que originaram os itens das duas escalas. Para completar o processo de desenvolvimento da escala, foi realizada uma análise de juízes, que contou com a participação de sete especialistas que avaliaram a pertinência dos itens para a mensuração do construto, bem como apontaram sugestões para a redação dos itens. Foi estabelecido o critério de concordância de $80 \%$ dos juízes para a manutenção dos itens das escalas.

\section{Etapa 2 - Verificação de propriedades psicométricas da Escala}

\section{Participantes}

Para a análise das evidências de validade psicométrica, as duas escalas foram aplicadas simultaneamente a um conjunto de 674 profissionais do Distrito Federal e dos estados do Rio de Janeiro e Minas Gerais. Adotou-se uma amostra de conveniência, com aplicação dos questionários a profissionais de diferentes organizações e setores. Foram estabelecidos dois critérios para inclusão do participante, a saber: (i) tempo mínimo de dois anos de trabalho, uma vez que uma das escalas testadas intencionava mostrar a visão evolutiva do desenvolvimento profissional; e (ii) concordância em assinar o termo de consentimento livre e esclarecido.

A pesquisa incluiu algumas questões a fim de permitir a descrição das variáveis sociodemográficas e funcionais dos pesquisados, as quais foram utilizadas para apontar diferenças entre grupos e correlações com a variável percepção do desenvolvimento profissional. As variáveis incluídas com esse objetivo foram as seguintes: sexo, idade, tempo de trabalho na organização atual e desde que começou a trabalhar, cargo (gerentes e não gerentes), ocorrência de promoção funcional recente (promovidos e não promovidos nos últimos três anos), grau de escolaridade (ensino fundamental, ensino médio, graduação e pós-graduação), tipo de organização (pública, privada e terceiro setor) e setor da economia (primário, secundário e terciário).
A atuação profissional deles divide-se em organizações públicas $(48,5 \%)$, privadas $(47,4 \%)$ ou do terceiro setor $(4,1 \%)$, sendo a maioria do setor terciário da economia $(84,9 \%)$. A amostra foi caracterizada por $50,5 \%$ de homens. A idade média dos pesquisados foi de 35,2 anos ( $\mathrm{dp}=11,5$ anos), tempo de trabalho médio de 14,9 anos ( $\mathrm{dp}=11,3$ anos). Em média, os participantes da pesquisa já trabalharam em 3,8 organizações $(\mathrm{dp}=2,4)$, sendo $82,3 \%$ não ocupantes de cargo gerencial, 49,6\% vinculados a grandes empresas, 44,8\% com ensino médio, $28,3 \%$ com graduação e $21,7 \%$, pós-graduação.

\section{Procedimentos}

Para a coleta de dados, os trabalhadores foram contatados em seu local de trabalho e convidados a participar da pesquisa de maneira voluntária. A forma de aplicação foi individual, com uso de questionário impresso. Foram esclarecidos os objetivos da pesquisa e garantido o anonimato dos participantes. Os questionários eram entregues aos participantes e recolhidos após o preenchimento. Vale registrar que o projeto desta pesquisa foi submetido a um Comitê de Ética, tendo a coleta de dados sido iniciada após o recebimento do parecer de aprovação do referido Comitê.

Todas as análises foram feitas utilizando o software IBM SPSS Statistics, versão 21.0., incluindo a análise paralela, a qual é processada no referido pacote estatístico a partir de uma programação RanEigen, que gera os eigenvalues aleatórios, de acordo com o tamanho da amostra e o número de itens da escala (Enzmann, 1997). Para a análise de dados, inicialmente utilizaram-se medidas de tendência central e dispersão, que permitiram caracterizar a amostra e compreender o comportamento das variáveis do estudo. A seguir, investigou-se a fatorabilidade da matriz de dados. Posteriormente, análise fatorial com extração dos eixos principais, assim como análise paralela foram os procedimentos adotados para definir e extrair o número de fatores considerado adequado. Por fim, o cálculo do alfa de Cronbach e lambda 2 de Guttman foram os procedimentos seguidos para aferir a confiabilidade interna dos fatores retidos.

\section{Resultados}

\section{Escala de Percepção Atual do Desenvolvimento Profissional - EPADP}

A Escala de Percepção Atual do Desenvolvimento Profissional foi constituída de oito afirmações associadas a uma escala do tipo likert de concordância de 11 
pontos, variando de zero, discordo totalmente, a 10, concordo totalmente. A análise de juízes manteve os oito itens inicialmente construídos para a escala, porém, com alguns ajustes de linguagem.

Para a verificação das propriedades psicométricas, inicialmente, foi investigada a fatorabilidade da medida mediante a inspeção da matriz de correlações, o cálculo do índice de adequação da amostra (KMO) e o teste de esfericidade de Bartlett. A realização desses procedimentos mostrou resultados satisfatórios uma vez que foi identificada presença de correlações significativas entre todos os itens da escala, o KMO foi de 0,82 e o teste de esfericidade de Bartlett significativo, $\left.\mathrm{X}^{2}=1.910,9 ; \mathrm{p}<0,00\right)$.

A seguir, identificou-se que a matriz de dados permitia extrair até um fator pelo critério de Kaiser (K1), que demanda autovalores (eigenvalues) maiores que 1. Esse resultado foi confirmado pelo método de análises paralelas (valor do autovalor empírico de 3,8 maior que o randômico de 1,2), que apontou a existência de um fator, assim como o gráfico de scree plot. O fator único extraído condensou $47,7 \%$ da variância do fenômeno e a retirada de qualquer um dos itens não se mostrou vantajosa.

A estrutura final sugerida para a escala ficou, portanto, com oito itens, sendo que as cargas fatoriais variaram de 0,55 a 0,78 e os coeficientes de precisão foram de 0,82 (alfa de Cronbach) e 0,83 (lambda 2 de Guttman). A Tabela 1 resume os resultados obtidos na análise fatorial da Escala de Percepção Atual do Desenvolvimento Profissional, na amostra da pesquisa.

Tendo em vista a identificação de evidências de validade da medida, foram realizadas algumas comparações entre os grupos constitutivos da amostra, visando demonstrar as informações que podem ser derivadas da medida ora relatada. Para tanto, procedeu-se à realização de análises de variância. A comparação dos valores da média aritmética de acordo com o sexo, o cargo (gerentes e não gerentes), a ocorrência de promoção funcional recente (promovidos e não promovidos nos últimos três anos), o grau de escolaridade (ensino fundamental, ensino médio, graduação e pós-graduação), o tipo de organização (pública, privada e terceiro setor) e o setor da economia (primário, secundário e terciário) mostra poucas diferenças (Tabela 2).

$\mathrm{Da}$ análise dos escores médios das diferentes categorias, destacam-se as seguintes diferenças significativas: (a) os trabalhadores do setor industrial percebem médias mais baixas de desenvolvimento profissional (média $=7,76, \mathrm{dp}=1,37$ ) do que aqueles que atuam no setor terciário da economia [média $=8,29$, $\mathrm{d} p=1,27, \mathrm{~F}(2,611)=6,22, \mathrm{p}=0,01]$; (b) quem tem pós-graduação [média $=8,43, \mathrm{dp}=1,08, \mathrm{~F}(3,639)=1,60$, $\mathrm{p}=0,19]$ percebe um desenvolvimento profissional atual ligeiramente mais elevado do que as pessoas que não têm (média $=8,16, \mathrm{dp}=1,34)$; e (c) quem exerce função de gerente (média $=8,44, \mathrm{dp}=1,34$ ) apresenta média de percepção de desenvolvimento profissional atual um pouco maior do que quem não exerce [média $=8,18$, $\mathrm{dp}=1,28, \mathrm{t}(638,2)=2,17, \mathrm{p}=0,03]$.

Ao analisar a magnitude dos desvios padrão desses grupos em relação aos valores das médias aritméticas respectivas, observa-se uma baixa dispersão (coeficiente de variação $<17 \%$ ), o que significa dizer que as pessoas que compõem cada categoria comparada tendem a ter visões compartilhadas em relação ao seu desenvolvimento, razão pela qual o valor da média aritmética representa bem o grupo. Isso revela, paralelamente, a

Tabela 1. Resultados da análise fatorial da EPADP

\begin{tabular}{lcc}
\hline Itens & Comunalidades & $\begin{array}{c}\text { Cargas } \\
\text { fatoriais }\end{array}$ \\
\hline 1. Atualmente domino todas as habilidades necessárias para a realização do meu trabalho. & 0,42 & 0,65 \\
2. Minha chefia já fez elogios sobre o meu desenvolvimento como profissional. & 0,31 & 0,55 \\
3. Atualmente, sinto-me bem preparado para realizar as atividades que me são destinadas. & 0,47 & 0,69 \\
4. Tive um expressivo desenvolvimento profissional desde que comecei a trabalhar. & 0,51 & 0,72 \\
5. Meus colegas elogiam meu crescimento profissional. & 0,33 & 0,58 \\
6. Com meus conhecimentos atuais, consigo realizar satisfatoriamente o meu trabalho. & 0,61 & 0,78 \\
7. Tenho me tornado um profissional mais qualificado. & 0,61 & 0,78 \\
8. Considero que meu desempenho como profissional tem melhorado. & 0,56 & 0,75 \\
\hline
\end{tabular}

Variância explicada $=47,7 \%$ - Alfa de Cronbach=0,82 - Lambda 2 de Guttman=0,83

Psico-USF, Bragança Paulista, v. 19, n. 1, p. 73-85, jan./abril 2014 
Tabela 2. Diferenças de média da Escala EPADP para as variáveis sociodemográficas.

\begin{tabular}{|c|c|c|c|c|c|}
\hline Variáveis & Condição & $\mathrm{N}$ & Média & Desvio padrão & Teste t/ANOVA \\
\hline \multirow[t]{2}{*}{ Cargo } & Gerente & 115 & 8,44 & 1,34 & \multirow{2}{*}{$\begin{array}{c}\mathrm{t}=2,00 ; \mathrm{gl}=638 ; \\
\mathrm{p}<0,05\end{array}$} \\
\hline & Não-Gerente & 525 & 8,18 & 1,28 & \\
\hline \multirow{2}{*}{$\begin{array}{l}\text { Promoção recente } \\
\text { (últimos } 3 \text { anos) }\end{array}$} & Promovido & 273 & 8,28 & 1,33 & \multirow{2}{*}{$\begin{array}{c}\mathrm{t}=0,84 ; \mathrm{gl}=631 ; \\
\mathrm{p}<0,40\end{array}$} \\
\hline & Não-promovido & 360 & 8,19 & 1,26 & \\
\hline \multirow[t]{2}{*}{ Sexo } & Feminino & 317 & 8,29 & 1,26 & \multirow{2}{*}{$\begin{array}{c}\mathrm{t}=1,07 ; \mathrm{gl}=635 ; \\
\mathrm{p}<0,28\end{array}$} \\
\hline & Masculino & 320 & 8,18 & 1,29 & \\
\hline \multirow[t]{4}{*}{ Escolaridade } & Ensino fundamental & 25 & 8,15 & 1,32 & \multirow{4}{*}{$\begin{array}{c}\mathrm{F}=1,16 ; \mathrm{gl}=639 ; \\
\mathrm{p}<0,19\end{array}$} \\
\hline & Ensino médio & 282 & 8,18 & 1,36 & \\
\hline & Graduação & 191 & 8,14 & 1,32 & \\
\hline & Pós-graduação & 142 & 8,43 & 1,08 & \\
\hline \multirow[t]{3}{*}{ Tipo de organização } & Pública & 309 & 8,26 & 1,19 & \multirow{3}{*}{$\begin{array}{c}\mathrm{F}=1,56 ; \mathrm{gl}=633 ; \\
\mathrm{p}<0,21\end{array}$} \\
\hline & Privada & 301 & 8,14 & 1,41 & \\
\hline & Terceiro setor & 24 & 8,58 & 0,93 & \\
\hline \multirow[t]{3}{*}{ Setor da economia } & Agropecuário & 5 & 8,50 & 0,52 & \multirow{3}{*}{$\begin{array}{c}\mathrm{F}=6,22 ; \mathrm{gl}=611 ; \\
\mathrm{p}<0,01\end{array}$} \\
\hline & Industrial & 85 & 7,76 & 1,37 & \\
\hline & De serviços & 522 & 8,29 & 1,27 & \\
\hline
\end{tabular}

pertinência de tecer conclusões relativas aos grupos comparados e demonstra, dessa forma, a utilidade da escala enquanto instrumento de diagnóstico do desenvolvimento grupal e não somente como ferramenta de avaliação de desenvolvimento individual.

\section{Escala de Percep̧ão Evolutiva do Desenvolvimento Profissional - EPEDP}

A Escala de Percepção Evolutiva do Desenvolvimento Profissional - EPEDP ficou composta por 13 itens, associados a uma escala de respostas de 11 pontos, na qual zero significa "nenhum preparo" e 10 revela que o profissional se considera "totalmente preparado". Nesta escala, o respondente é solicitado a oferecer duas respostas a cada item: uma para a sua percepção de preparo quando começou a trabalhar e outra para o grau de preparo atual. Assim, o escore de cada item é calculado a partir da subtração dos valores das respostas relativas ao desenvolvimento profissional no momento de início da carreira daqueles valores apontados como de desenvolvimento atual, ou seja, valores atuais menos valores iniciais. A análise de juízes apontou que alguns itens da escala deveriam indicar comportamentos mais facilmente identificáveis pelos respondentes, sendo, portanto, aperfeiçoada a redação dos mesmos, mas sem aumento no número de itens, que foi considerado exaustivo para a indagação do fenômeno focalizado.
A avaliação dos indicadores psicométricos mostrou condições adequadas de fatorabilidade da matriz de dados. O valor do KMO para esta escala foi de 0,94, o teste de esfericidade de Bartlett significativo, $\mathrm{X}^{2}=4.963,5 ; \mathrm{p}<0,00$ e a inspeção da matriz de correlações revelou correlações significativas positivas entre os itens.

A definição do possível número de fatores a extrair foi realizada considerando-se o critério de Kaiser (K1), a análise paralela e inspeção visual do gráfico de Scree Plot. A possibilidade de extração de até um fator da matriz de dados foi pelo resultado obtido no critério de Kaiser (K1), que demanda autovalores (eigenvalues) maiores que 1. Esse resultado foi confirmado pelo método de análise paralela (valor do autovalor empírico de 7,5 maior que o randômico de 1,1), confirmando a existência de um fator, assim como o gráfico de scree plot. O fator único apresentado foi capaz de explicar $57,9 \%$ da variância.

Os 13 itens permaneceram na estrutura final sugerida para a escala, sendo que as cargas fatoriais variaram de 0,62 a 0,84 e os coeficientes de precisão foram de 0,94 (alfa de Cronbach e também lambda 2 de Guttman). A Tabela 2 apresenta os resultados obtidos na análise fatorial da Escala de Percepção Evolutiva do Desenvolvimento Profissional. A retirada de qualquer um dos itens não trouxe ganhos à 
confiabilidade da medida, o que confirma a pertinência de manter todos eles.

Em relação aos escores obtidos na Escala de Percepção Evolutiva do Desenvolvimento Profissional - EPEDP, considerando que eles resultam da subtração da resposta de desenvolvimento quando a pessoa iniciou a sua experiência de trabalho do escore do desenvolvimento atual, os valores resultantes poderiam variar de -10 (percepção de desenvolvimento atual $=0$ e percepção de desenvolvimento inicial $=$ 10) a +10 (percepção de desenvolvimento atual $=10$ e percepção de desenvolvimento inicial=0). A comparação das médias de acordo com o sexo, o cargo, a ocorrência de promoção funcional recente, o grau de escolaridade, o tipo de organização e o setor da economia mostra poucas diferenças entre as médias (Tabela 4). Merece maior destaque na comparação dessas médias a diferença de quem foi promovido nos últimos 3 anos $(\mathrm{t}(2,614)=3,32 ; \mathrm{p}=0,01)$ que percebe uma evolução maior do desenvolvimento profissional (média $=2,70, \mathrm{dp}=1,79)$ do que quem não foi promovido (média $=2,21, \mathrm{dp}=1,85$ ).

No caso das derivações que podem resultar da utilização da escala EPEDP, vale destacar que os grupos com promoções recentes se descrevem como tendo tido mais desenvolvimento que os demais. Ainda assim, observa-se grande discrepância nas respostas dadas pelos grupos, constatada ao analisar a relação entre o tamanho do desvio padrão $(1,79)$ e o valor da média aritmética do grupo (2,70), que resulta em um coeficiente de variação de 62,9\% (de discrepância) Esse dado demonstra que as percepções não são similares, o que permite afirmar que apesar das diferenças significativas entre grupos, alguns dos respondentes com promoções mais recentes não percebem evolução no seu grau de desenvolvimento.

\section{Correlações da EPADP e da EPEDP}

Seria esperado que a idade, o tempo de trabalho e o número de organizações nas quais as pessoas já trabalharam apresentassem alguma correlação com o desenvolvimento profissional, uma vez que tal conceito pressupõe um processo gradual de aquisição de competências laborais advindas das experiências de trabalho. Considerando as diferenças entre as escalas, esperava-se que a EPEDP tivesse correlações mais altas com essas variáveis, uma vez que ela possibilita uma comparação temporal, a partir das respostas para o momento inicial de carreira e o momento atual. Considerando essa especificidade da EPEDP, optou-se por realizar a correlação não apenas com o escore resultante da diferença entre o valor final e inicial do desenvolvimento profissional, mas também para os valores apontados como iniciais e finais.

Quando relacionamos os resultados da EPADP e da EPEDP com a idade, o tempo de trabalho (total e na organização atual), o número de organizações nas quais os participantes da pesquisa já trabalharam, observa-se

Tabela 3. Resultados da análise fatorial da EPEDP

\begin{tabular}{lcc}
\hline Itens & Comunalidades & Cargas fatoriais \\
\hline Realizar as atividades de forma autônoma & 0,38 & 0,62 \\
Planejar o trabalho & 0,67 & 0,82 \\
Executar as tarefas demandadas & 0,57 & 0,75 \\
Tomar decisão na realização das tarefas & 0,74 & 0,86 \\
Trabalhar em equipe & 0,49 & 0,70 \\
Fazer autoavaliação do seu desempenho & 0,69 & 0,83 \\
Propor melhorias & 0,70 & 0,84 \\
Demonstrar conhecimento técnico & 0,66 & 0,81 \\
Trabalhar sozinho & 0,58 & 0,76 \\
Interagir com os colegas & 0,56 & 0,75 \\
Atuar nos diferentes processos de trabalho & 0,66 & 0,81 \\
Ter atitudes adequadas ao ambiente de trabalho & 0,45 & 0,67 \\
Interagir com a chefia & 0,40 & 0,63 \\
\hline
\end{tabular}

Variância explicada $=57,9 \%$ - Alfa de Cronbach=0,94 - Lambda 2 de Guttman=0,94 
Tabela 4. Diferenças de média da Escala EPEDP para as variáveis sociodemográficas

\begin{tabular}{|c|c|c|c|c|c|}
\hline Variáveis & Condição & $\mathrm{N}$ & Média & Desvio padrão & Teste t/ANOVA \\
\hline \multirow[t]{2}{*}{ Cargo } & Gerente & 111 & 2,60 & 1,86 & \multirow{2}{*}{$\begin{array}{c}\mathrm{t}=1,15 ; \mathrm{gl}=620 ; \\
\mathrm{p}<0,25\end{array}$} \\
\hline & Não-Gerente & 511 & 2,38 & 1,83 & \\
\hline \multirow{2}{*}{$\begin{array}{l}\text { Promoção recente } \\
\text { (últimos } 3 \text { anos) }\end{array}$} & Promovido & 274 & 2,70 & 1,79 & \multirow{2}{*}{$\begin{array}{c}\mathrm{t}=3,32 ; \mathrm{gl}=614 ; \\
\mathrm{p}<0,01\end{array}$} \\
\hline & Não-promovido & 342 & 2,21 & 1,85 & \\
\hline \multirow[t]{2}{*}{ Sexo } & Feminino & 306 & 2,51 & 1,98 & \multirow{2}{*}{$\begin{array}{c}\mathrm{t}=1,09 ; \mathrm{gl}=618 ; \\
\mathrm{p}<0,28\end{array}$} \\
\hline & Masculino & 314 & 2,35 & 1,68 & \\
\hline \multirow[t]{4}{*}{ Escolaridade } & Ens. fundamental & 22 & 2,39 & 1,51 & \multirow{4}{*}{$\begin{array}{c}\mathrm{F}=0,29 ; \mathrm{gl}=621 ; \\
\mathrm{p}<0,83\end{array}$} \\
\hline & Ensino médio & 274 & 2,38 & 1,82 & \\
\hline & Graduação & 185 & 2,54 & 1,97 & \\
\hline & Pós-graduação & 141 & 2,39 & 1,71 & \\
\hline \multirow[t]{3}{*}{ Tipo de organização } & Pública & 305 & 2,51 & 1,73 & \multirow{3}{*}{$\begin{array}{c}\mathrm{F}=0,86 ; \mathrm{gl}=616 ; \\
\mathrm{p}<0,42\end{array}$} \\
\hline & Privada & 291 & 2,36 & 1,92 & \\
\hline & Terceiro setor & 21 & 2,07 & 1,78 & \\
\hline \multirow[t]{3}{*}{ Setor da economia } & Agropecuário & 5 & 3,06 & 0,71 & \multirow{3}{*}{$\begin{array}{c}\mathrm{F}=0,43 ; \mathrm{g}=596 ; \\
\mathrm{p}<0,65\end{array}$} \\
\hline & Industrial & 87 & 2,50 & 1,97 & \\
\hline & De serviços & 505 & 2,39 & 1,83 & \\
\hline
\end{tabular}

que a EPADP não apresenta correlação significativa $(\mathrm{p}<0,05)$ com nenhuma dessas variáveis. Esse resultado pode representar uma limitação da medida, uma vez que seria esperado que a idade, o tempo de trabalho e o número de organizações nas quais as pessoas já trabalharam apresentassem alguma correlação com o desenvolvimento profissional, uma vez que tal conceito pressupõe um processo gradual de aquisição de competências laborais resultantes das experiências de trabalho. Nesse sentido, a ausência de correlação entre essas variáveis pode indicar uma fragilidade da referida medida em discriminar níveis distintos de desenvolvimento profissional.

Em relação à EPEDP, os resultados mostram que a escala não apresenta correlação com a idade ou com o tempo de trabalho total, mas apresenta correlação com o tempo de trabalho na organização atual, com o número de funcionários da organização e com o número de organizações nas quais a pessoa já trabalhou. Esse resultado mostra que, por um lado, quando as pessoas trabalham há mais tempo na mesma organização e em organizações com mais funcionários, elas tendem a perceber maior ganho ou desenvolvimento, ao analisar o seu grau de preparo profissional quando começaram a trabalhar e o seu grau de preparo atual. Por outro lado, quando a pessoa trocou de organização mais vezes, ela tende a perceber menor diferença entre o seu grau de preparo atual e o grau de preparo inicial. Porém, vale destacar que a medida do desenvolvimento profissional atual na escala EPEDP foi a que apresentou o maior número de correlações com as variáveis sociodemográficas e funcionais (Tabela 5).

Foram também analisadas as correlações entre as duas medidas considerando, em relação à EPEDP, tanto os escores de desenvolvimento inicial e desenvolvimento atual como o escore de diferença entre eles. A EPADP apresentou correlação baixa com o desenvolvimento inicial apontado pela $\operatorname{EPEDP}(r=0,28$; $p=0,01)$, correlação alta com o desenvolvimento final da EPEDP $(r=0,61 ; p=0,01)$ e ausência de correlação com o escore da diferença entre o desenvolvimento final e o inicial da EPEDP $(r=0,03 ; p=0,40)$. Essas análises permitem afirmar que a quantidade de variância compartilhada não supera $38 \%$ em qualquer um dos casos, sendo de apenas $9 \%$ quando comparadas as medidas EPADP e EPEDP.

\section{Discussão}

Em primeiro lugar, um aspecto importante no processo de construção e investigação das evidências de validade das escalas EPADP e EPEDP consiste na comparação realizada em relação às duas medidas. Observa-se que elas não apresentam correlação 
Tabela 5. Correlações da EPADP e EPEDP com variáveis pessoais e funcionais

\begin{tabular}{|c|c|c|c|c|c|c|c|c|}
\hline & 2 & 3 & 4 & 5 & 6 & 7 & 8 & 9 \\
\hline 1. DP atual (EPEDP) & $0,46 * *$ & $0,61 * *$ & 0,06 & $0,15^{* *}$ & $0,14^{* *}$ & $0,10^{*}$ & $0,09 *$ & $-0,16^{* *}$ \\
\hline 2. DP inicial (EPEDP) & & $0,28^{* *}$ & $-0,86^{* *}$ & $0,12 * *$ & $0,09 *$ & $-0,04$ & $0,14 * *$ & $-0,16^{* *}$ \\
\hline 3. EPADP & & & 0,03 & 0,03 & 0,04 & 0,03 & 0,02 & $-0,04$ \\
\hline 4. EPEDP & & & & $-0,05$ & $-0,02$ & $0,11 * *$ & $-0,10^{*}$ & 0,08 \\
\hline 5. Idade & & & & & $0,87^{* *}$ & $0,67 * *$ & $0,28 * *$ & $0,15^{* *}$ \\
\hline 6. Tempo trabalho total & & & & & & $0,69 * *$ & $0,30 * *$ & $0,20 * *$ \\
\hline 7. Tempo trabalho organização & & & & & & & $-0,11 * *$ & $0,19 * *$ \\
\hline 8. $\mathrm{N}^{\mathrm{o}}$ organizações que trabalhou & & & & & & & & 0,06 \\
\hline 9. $\mathrm{N}^{\circ}$ funcionários da organiz. & & & & & & & & \\
\hline
\end{tabular}

significativa, o que revela que, de fato, avaliam construtos distintos, com possibilidades de uso diferenciados. Ainda assim, cabe destacar que observou-se correlação positiva de baixa magnitude, considerando a classificação de Miles e Shevlin (2001) entre a EPADP e a percepção inicial de desenvolvimento profissional da EPEDP e correlação positiva de alta magnitude entre a EPADP e a percepção atual de desenvolvimento profissional da escala EPEDP. A respeito dessas correlações entre a EPADP e a EPEDP, cabe assinalar que esses dados ainda são exploratórios e, por isso, devem ser interpretados cuidadosamente, uma vez que as duas medidas foram desenvolvidas recentemente e não apresentam evidências prévias de validade.

A análise dos dados deste estudo exploratório evidenciam que a percepção atual de desenvolvimento compartilha mais variância com a percepção de evolução do desenvolvimento que ele tem no momento atual se comparado com a magnitude da variância compartilhada com a percepção de evolução do desenvolvimento que tinha no início da sua carreira. Essa maior variância entre EPADP e a percepção final de desenvolvimento da EPEDP já seria esperada uma vez que ambas se referem ao nível atual de desenvolvimento das pessoas. Contudo, a ausência de correlação entre a EDADP e a EDEDP, mostra que ambos os construtos são teórica e empiricamente diferenciáveis. Por essa razão, é defendida a pertinência de serem tratadas como medidas efetivamente distintas.

Ainda sobre a magnitude das correlações encontradas, valor mais elevado e negativo é visto em relação à avaliação de desenvolvimento inicial com o escore da EPEDP. Esse resultado é favorável à medida e esperado, uma vez que está sendo defendido que a percepção evolutiva do desenvolvimento profissional seria o resultado da subtração da percepção do desenvolvimento profissional atual pela percepção do desenvolvimento profissional inicial. Logo, essas variáveis são teoricamente relacionadas, o que justifica a magnitude alta (de acordo com Miles \& Shevlin, 2001) da relação encontrada. Entretanto, o valor observado da correlação $(-0,86)$ paralelamente evidencia que embora essas duas variáveis compartilhem $73,9 \%$ da variância, ainda restam $26 \%$ de variância que são específicas de cada fenômeno.

Ainda sobre essas correlações vale discutir o fato de a EPEDP não apresentar correlação significativa com os escores da avaliação atual de desenvolvimento profissional que compõem essa medida. Este resultado é importante na medida em que ele confirma que a escala, de fato, apresenta uma visão evolutiva do crescimento profissional sem influência direta da percepção atual do grau de desenvolvimento em que a pessoa se encontra.

Uma análise conjunta das variáveis que se relacionam com as duas escalas de desenvolvimento profissional mostra que, enquanto a escala de percepção atual está relacionada com o setor da economia, a escolaridade e o exercício de função gerencial, a escala de percepção evolutiva relaciona-se ao exercício de função gerencial, à ocorrência de promoção recente (nos últimos três anos), ao número de organizações nas quais a pessoa já trabalhou, ao tempo de vínculo com a organização de trabalho atual e ao número de funcionários da organização de vínculo atual do trabalhador. Contudo, vale destacar que a percepção de desenvolvimento profissional atual da EPEDP apresenta mais correlações com as variáveis sociodemográficas 
e funcionais do que a medida do escore de ganho do desenvolvimento profissional. Esse resultado sinaliza para a possibilidade de se considerar apenas o valor final do desenvolvimento profissional nas pesquisas, utilizando os valores iniciais apenas como artifício para que o participante da pesquisa leve em consideração essa visão evolutiva de seu desenvolvimento profissional. Esse resultado também pode sinalizar que a explicação do desenvolvimento profissional talvez esteja mais relacionada com outras variáveis do indivíduo ou com variáveis do contexto.

Como pode ser visto, quase todas as variáveis relacionam-se com uma ou com outra variável, sendo que a escala de percepção evolutiva do desenvolvimento profissional apresenta relação com maior número de variáveis do que a escala que mensura percepção atual. $O$ fato de a EPEDP estar positivamente relacionada com o número de empregados da organização em que os pesquisados trabalham corrobora os achados de pesquisas estrangeiras (Elbadri, 2001; Wong, Marshall \& Thwaites, 1997) e nacionais (Mourão, 2009), que mostram que organizações com mais funcionários oferecem maiores possibilidades de qualificação para a sua mão de obra.

Por outro lado, o fato de nenhuma das duas escalas de desenvolvimento profissional apresentar relação com o tipo de organização (se pública, privada ou do terceiro setor), vai de encontro aos achados de Mourão (2009) que constatou que as organizações públicas oferecem mais oportunidade de qualificação profissional que as organizações privadas. Seria preciso, portanto, a replicação do estudo em outras amostras, além de estudos que investigassem a razão de os servidores públicos não perceberem maior desenvolvimento profissional, mesmo o serviço público oferecendo mais oportunidades de qualificação.

No que diz respeito especificamente às propriedades da medida de desenvolvimento profissional, cabe destacar que as evidências encontradas mostram a pertinência das decisões tomadas. Por um lado, observa-se que o desenvolvimento é um processo contínuo que permite ao indivíduo perceber o seu crescimento vivenciado ao longo do tempo (Paquay e colaboradores, 2012). Isso possibilita a identificação de respostas diferenciadas para ambas as medidas ora apresentadas. Falar sobre desenvolvimento profissional, dessa forma, tanto permite fazer referência a um estado atual (quanto desenvolvimento pessoal percebo) como permite focar no processo de transformação (quanto mudei ao longo do tempo, em termos de crescimento profissional). A importância decorrente dessa distinção está nas possibilidades de aplicação prática dessas informações. Por um lado, gestores organizacionais, orientadores profissionais, planejadores de carreira e profissionais de áreas afins podem ter interesse em saber o estado de desenvolvimento atual percebido por determinados indivíduos ou grupos. Por outro lado, também pode haver interesse em indagar, por exemplo, o desenvolvimento percebido ao longo do tempo como resultado da implementação de determinados projetos ou estratégias organizacionais ou de decisões relativas a planejamentos de carreira. Nesse caso, a medida de percepção de evolução seria a mais adequada.

Também pode ser concluído, a partir dos resultados observados, que as escalas permitem capturar diferenças entre grupos da organização. Assim, em termos práticos podem ser realizados diagnósticos por grupos sobre o grau de desenvolvimento, comparando, por exemplo, diferentes áreas da organização. Perante a ausência de diferenças significativas, o gestor poderá concluir que a vinculação dos colaboradores a um ou a outro grupo não está associada à percepção de desenvolvimento.

Por fim, de posse das evidências encontradas, torna-se pertinente afirmar que as escalas EPADP e EPEDP são ferramentas úteis para o diagnóstico em organizações. O processo de desenvolvimento das mesmas mostrou-se adequado, uma vez que a investigação das evidências de validade revelou resultados satisfatórios. Diante do exposto, considera-se relevante oferecer essas medidas como instrumentos de pesquisa e ação gerencial que permitem preencher, ainda que parcialmente, a lacuna identificada no campo, possibilitando avanço dos estudos da área ao contar com medidas confiáveis, que tornam viável a comparabilidade de resultados de pesquisa que venham a adotá-las.

\section{Considerações finais}

A investigação das evidências de validade das escalas de desenvolvimento profissional pode fornecer aos pesquisadores da área um instrumento útil para diagnosticar a percepção que os trabalhadores têm de seu desenvolvimento profissional. Os resultados apontam para estruturas fatoriais estáveis, sendo possível recomendar o uso de ambas as escalas. A escolha de qual delas utilizar deve ser feita considerando-se a informação central desejada. Por exemplo, se a pesquisa pretende avaliar o quanto a pessoa se desenvolveu desde 
que começou a trabalhar, o ideal seria usar a escala que adota a percepção evolutiva. Nesse caso, pode-se também variar o comando da escala e mensurar o grau de desenvolvimento profissional desde que o funcionário começou a trabalhar em uma determinada empresa. Essa pode ser uma informação bastante útil para os profissionais de gestão de pessoas. Por outro lado, se a intenção for avaliar o quanto de desenvolvimento profissional os trabalhadores avaliam que possuem num dado momento, a escala com a percepção atual seria mais indicada.

Comparando-se as duas escalas observa-se que, por um lado, a escala de percepção atual do desenvolvimento profissional tem um processo de aplicação mais simples e um número menor de itens. Por outro lado, a escala de percepção atual do desenvolvimento profissional tem uma estrutura mais estável (com valores mais elevados das cargas fatoriais e também do grau de precisão da escala). A correlação entre as escalas indica que elas medem aspectos diferentes do desenvolvimento profissional e, portanto, têm objetivos diferentes, e cabe aos pesquisadores definir qual se aplica melhor ao seu interesse de pesquisa. Contudo, como este foi o primeiro estudo com essas medidas, é importante que pesquisas em novas amostras indiquem outras evidências de validade dessas escalas e testem novamente a correlação entre as mesmas, para confirmar ou não os resultados aqui descritos.

Como limitações do presente estudo podem ser apontadas: (i) a coleta de dados ter se limitado geograficamente ao Distrito Federal e a dois outros estados da região Sudeste; (ii) a ausência de avaliação da estabilidade temporal da escala; (iii) a análise de correlação das medidas exclusivamente com variáveis sociodemográficas e funcionais. Como há poucos estudos na literatura nacional e estrangeira sobre escalas de percepção de desenvolvimento profissional, sugerem-se novos estudos com essas escalas, relacionando-as a outros construtos sobretudo da área de comportamento organizacional, a fim de verificar o quanto a medida poderia ser útil para aumentar a compreensão dos fenômenos organizacionais. Também sugere-se a replicação desse estudo em amostra nacional. Para a avaliação da estabilidade temporal, sugere-se que a aplicação e reaplicação do instrumento ocorra em um espaço curto de tempo, uma vez que um dos propósitos da medida é justamente mensurar os ganhos de desenvolvimento profissional ao longo do tempo.

Conclui-se que ambas as escalas apresentadas podem contribuir não apenas para diagnosticar qual a percepção de desenvolvimento profissional, mas também para investigar a existência de correlações com outros atributos relativos a comportamentos organizacionais ou às práticas de gestão de pessoas. Elas também podem ser medidas importantes para os profissionais que atuam com orientação profissional e planejamento de carreira.

\section{Referências}

Abbad, G., Zerbini, T., \& Souza, D. B. L. (2010). Panorama das pesquisas em educação a distância no Brasil. Estudos de Psicologia (UFRN), 15, 291-298.

Aguinis, H., \& Kraiger, K. (2009). Benefits of training and development for individuals and teams, organizations, and society. Annual Review of Psychology, 60, 451-474.

Antonello, C. S. (2005). Articulação da aprendizagem formal e informal: seu impacto no desenvolvimento de competências gerenciais. Alcance, 12(2), 183-209.

Arthur, M. B., \& Rousseau, D. (Orgs.) (1996). The boundaryless career: individual action and economic change. Nova Iorque: Oxford University Press.

Bardin, L. (1977). Análise de conteúdo. Lisboa. Portugal: Edições 70.

Baruch, Y. (2006). Career development in organizations and beyond: balancing traditional and contemporary viewpoints. Human Resource Management Review, 16(2), 125-38.

Bassanini, A., Booth, A., Brunello, G., De Paola, M., \& Leuven, E. (2005). Workplace training in Europe. Disponível em: http://www.iser.essex.ac.uk/epunet/2005. Acessado em: 2 março de 2008.

Bauer, M. W. (2010). Análise de conteúdo clássica: uma revisão. Em: M. W. Bauer \& G. Gaskell (Orgs.). pesquisa qualitativa com texto: imagem e som: um manual prático. (pp. 189-217). Petrópolis: Vozes.

Berkvens, J., Kalyanpur, M., Kuiper, W., \& Van den Akker, J. (2012). Improving adult learning and professional development in a post-conflict area: the case of Cambodia. International Journal of Educational Development, 32, 241-251.

Butteriss, M. (1999), Help wanted: the complete guide to HR for canadian entrepreneurs. Toronto: John Wiley\& Sons. 
Coelho Jr., F. A., \& Borges-Andrade, J. E. (2008). Uso do conceito de aprendizagem em estudos organizacionais. Paideia, 40(18), 221-234.

Coelho Jr., F. A., \& Mourão, L. (2011). Suporte à aprendizagem informal no trabalho: uma proposta de articulação conceitual. Revista de Administração da Mackenzie, 12(6), 224-253.

Conlon, T. J. (2004). A review of informal learning literature, theory and implications for practice in developing global professional competence. Journal of European Industrial Training, 28(2-4), 283-295.

Day, J. (2004). Évaluer la formation continue des enseignants dans le context d'une école en changement en vue de favoriser le développement professionnel des acteurs et d'améliorer leur efficacité? Em L. Paquay. L'évaluation des enseignants. Tensions et enjeux (pp. 87-119). Paris: l'Harmattan.

Elbadri, A. N. A. (2001). Training practices of Polish companies: an appraisal and agenda for improvement. Journal of European Industrial Training, 25, 69-79.

Enzmann, D. (1997). RanEigen: a program to determine the parallel analysis criterion for the number of principal components. Applied Psychological Measurement, 21, 232-247.

Flach, L., \& Antonello, C. S. (2011). Organizações culturais e a aprendizagem baseada em práticas. Cadernos da EBAPE.BR, 9(1), 155-175.

Freitas, I. A., \& Mourão, L. (2012). Medidas de impacto de TD\&E: o nível da mudança organizacional. Em G. S. Abbad, L. Mourão, P. P. M. Meneses; T. Zerbini; J. E. Borges-Andrade \& R. Dias (Orgs.). Medidas de avaliação em TD\&E. (pp. 163-176). Porto Alegre: Artmed.

Golding, L., \& Gray, I. (2006). Continuing professional development for clinical psychologists: a practical handbook. The British Psychological Society. Oxford: Blackwell Publishing.

Goldstein, I. L., \& Gilliam, P. (1990). Training system issues in the year 2000. American Psychologist, 45(2), 134-143.

Guskey, T. R. (2000). Evaluating professional development. Thousand Oaks: Sage Publications.

Hall, D. T. (2002). Careers in and out of organizations. Londres: Sage.
Jasper, M. (2006). Professional development, reflection, and decision-making. Oxford: Blackwell Publishing.

Livingstone, D. (1999). Exploring the icebergs of adult learning. Canadian Journal for the Study of Adult Education, 3(2), 49-72.

Malcolm, J., Hodkonson, P., \& Colley, H. (2003). The interrelationships between informal and formal learning. Journal of Workplace Learning, 15(7/8), 131-318.

Miles, J., \& Shevlin, M. (2001). Applying regression \& correlation. A guide for students and researches. London: Sage.

Mourão, L. (2009). Oportunidades de qualificação profissional no Brasil: reflexões a partir de um panorama quantitativo. Revista de Administração Contemporânea, 13(1), 136-153.

Mourão, L., \& Meneses, P. P. M. (2012). Medidas de impacto de TD\&E: o nível do valor final. Em G. S. Abbad, L. Mourão, P. P. M. Meneses, T. Zerbini, J. E. Borges-Andrade \& R. Dias (Orgs). Medidas de Avaliação em TD\&E. (pp. 177-188). Porto Alegre: Artmed.

Paquay, L., Wouters, P., \& van Nieuwenhoven, C. (2012). A avaliação, freio ou alavanca do desenvolvimento professional?. Em L. Paquay, P. Wouters, \& C. van Nieuwenhoven, C. (Orgs.). A avaliação como ferramenta do desenvolvimento profissional de educadores. (pp.13-39). Porto Alegre: Penso.

Portal da Transparência. Disponível em: http://www. portaltransparencia.gov.br. Acessado em: 18 abril 2012.

Rousseau, D. M. (1997). Organizational behavior in the new organizational era. Annual Review of Psychology, $48,515-546$.

Salas, E., \& Cannon-Bowers, J. (2001). The science of training: a decade of progress - Annual Review Psychology, 52, 471- 499.

SamGnanakkan, S. (2010). Mediating role of organizational commitment on HR practices and turnover intention among ICT professionals. Journal of $\mathrm{Ma}$ nagement Research, 10(1), 39-61.

Watkins, K. E., \& Marsick, V. J. (1992). Toward a Theory of informal and incidental learning in organizations. International Journal of Lifelong Education, 11(4), 287-300.

Psico-USF, Bragança Paulista, v. 19, n. 1, p. 73-85, jan./ abril 2014 
Wong, C., Marshall, J., \& Thwaites, A. (1997). Management training in small and medium-sized enterprises: methodological and conceptual issues. The International Journal of Human Resource Management, 8(1) 44-65.
Recebido em: 18/12/2012

Reformulado em: 10/10/2013

Segunda reformulação em: 23/10/2013

Aprovado em: 12/11/2013

Sobre as autoras:

Luciana Mourão é doutora em Psicologia pela Universidade de Brasília (UnB), professora do Programa de Pós-Graduação em Psicologia da Universidade Salgado de Oliveira e também da Associação Internacional de Educação Continuada - AIEC. Pesquisadora do CNPq, é participante dos grupos de pesquisa da Associação Nacional de Pesquisa e Pós-Graduação em Psicologia.

Katia Puente-Palacios é doutora em Psicologia na Universidade de Brasília (UnB), tendo realizado uma parte deste último na Groningen Rijksuniversiteit, na Holanda (2002). Em 2009, realizou pós-doutorado na Universidade de Valencia-Espanha. Atua como professora adjunta da UnB e é pesquisadora do CNPq.

Juliana Barreiros Porto é doutora em Psicologia pela Universidade de Brasília (UnB), professora adjunta do Departamento de Psicologia Social e do Trabalho e do Programa de Pós-Graduação em Psicologia Social, do Trabalho e das Organizações da UnB. É pesquisadora do CNPq, líder do Núcleo de Pesquisa em Cultura, Valores e Comportamento (CNPq). 
